\title{
Los biodatos como indicadores de la conducta vocacional universitaria: \\ Elaboración de un instrumento de evaluación
}

\author{
Esperanza Rocabert Beut, Adela Descals Tomás, \\ Amparo Gómez Artiga
}

Dpto. de Psicología Evolutiva y de la Educación, Universidad de Valencia

\section{España}

esperanza.rocabert@uv.es 


\section{Resumen}

Este trabajo se sitúa en el marco de un proyecto de investigación de carácter interuniversitario, subvencionado por el Ministerio de Ciencia y Tecnología, que trata de propiciar el conocimiento científico de la conducta vocacional y preprofesional de los estudiantes universitarios. Entre los distintos indicadores de la conducta vocacional considerados en dicha investigación, este estudio se centra en la elaboración de un cuestionario que analice los biodatos académicos (o datos biográficos dentro del área académica) relacionados con el desarrollo vocacional de los estudiantes. Es decir, se analizan las vivencias, hechos y conductas del estudiante en relación a su desarrollo vocacional para comprobar la adecuación o eficacia de las mismas a la hora de planificar y explorar sus opciones de especialización y preparación de su profesionalidad. A partir de la pertinente revisión teórica, de las aportaciones de diversos investigadores-expertos y de los resultados de distintos estudios desarrollados en el ámbito del proyecto de investigación, elaboramos el "Cuestionario de Biodatos Universitarios: CBU" que estaba configurado por 9 ítems agrupados en cinco apartados: la Motivación o Interés por la opcion universitaria escogida; la Toma de Decisiones Académicas o Preprofesionales (Optativas, Practicum); el Grado de Decisión sobre especialidad o salida profesional, la Experiencia Laboral y las Dificultades percibidas en el Proceso de Toma de Decisiones preprofesionales. Este cuestionario se aplicó a 2169 estudiantes universitarios para determinar su homogeneidad interna y su relación con el desarrollo de carrera eficaz. Los análisis estadísticos realizados muestran como el cuestionario es capaz de clasificar adecuadamente a estudiantes universitarios en función de su grado de desarrollo de carrera: desde eficaz a no eficaz.

Palabras Clave: asesoramiento vocacional, conducta vocacional, biodatos, enseñanza universitaria. 


\section{Introducción}

Este estudio se desarrolla en el marco del proyecto de investigación "Conducta vocacional y asesoramiento profesional de los estudiantes universitarios: Protocolo informático para la autoayuda preprofesional”, que ha sido subvencionado por el Ministerio de Ciencia y Tecnología (I+D BS02001-3150). Dicho proyecto de carácter interuniversitario, en el que han participado doce universidades españolas, se ha centrado en el análisis de la conducta vocacional y preprofesional de los estudiantes universitarios con el objetivo de que, posteriormente, se formulen los correspondientes planes de actuación para los servicios de asesoramiento universitarios. El proyecto ha sido dirigido por el profesor Rivas, quien viene mostrando su insistente trayectoria investigadora en esta temática.

La investigación llevada a cabo desde este proyecto ha permitido, a lo largo de tres estudios, la elaboración de un conjunto de instrumentos que facilitan el análisis de la conducta vocacional de los estudiantes universitarios. En una primera exploración inicial, se aplicó el Protocolo 2001 a 2470 universitarios que estaban finalizando sus estudios en todas las universidades colaboradoras en el proyecto. A partir de los resultados y de las aportaciones de los expertos que participaron en la investigación, se elaboró el Protocolo 2004 que se aplicó a 421 estudiantes en un estudio piloto realizado únicamente en tres de las Universidades participantes en la investigación (Valencia, Almería y Alicante). Los resultados de este segundo estudio permitieron la formulación del Protocolo 2005, instrumento que en un tercer estudio se aplicó a 2.169 estudiantes de las diferentes universidades que participaban en el proyecto.

El Protocolo 2005 permite la evaluación de distintos indicadores de la conducta vocacional universitaria: identidad vocacional, biodatos, factores vocacionales, estudio, ideas vocacionales, intereses profesionales, psicoemocionalidad y toma de decisiones para el desarrollo de carrera. Entre dichos indicadores, el trabajo que presentamos a continuación analiza los biodatos o datos biográficos referidos al ámbito académico universitario que se relacionan con una conducta vocacional universitaria eficiente. 
En un sentido amplio el término biodatos hace referencia al bagaje experiencial que el individuo aporta a la conducta vocacional con miras a su integración sociolaboral (Castaño 1983; Farmer 1985; O’Neil et al., 1980, Rocabert, 1995). La historia personal, las experiencias que configuran al individuo, desde siempre han tenido un determinado valor en el ámbito vocacional, llegando a expresarse como un principio general que: "El mejor predictor de la conducta futura es la conducta pasada "y la matización que se añade a lo anterior "....y lo que el individuo piensa de la misma".

Esa línea de trabajo engloba un conjunto de variables individuales que interactúan con el ambiente próximo. Tratadas en principio como variables demográficas y moduladoras de las diferencias muestrales en las investigaciones psicosociológicas, pasan a tener un peso específico cuando se las define como variables de la vida o biodatos (Owens, 1983) y se comprueba que su papel, no es sólo de modulación, sino netamente de predictor de una gran parte de la varianza explicada de la conducta vocacional de los individuos. Los biodatos los encontramos en los formularios de solicitud de trabajo, en las guías vocacionales y en cuestionarios de antecedentes; sus ítems son indicadores de hechos susceptibles de comprobación objetiva y subjetiva de ideas o sentimientos expresados sobre acontecimientos de la vida diaria, escolar o familiar. Son indicadores que hacen casi siempre referencia a hechos del presente y sobre todo, del pasado inmediato.

Aunque existe una gran variedad de biodatos posibles, en nuestro estudio nos hemos limitado a analizar sólo aquellas realizaciones que un sujeto puede aportar a modo de currículum vitae, que son objeto de evaluación, y que señalan tanto los límites como las potencialidades respecto al mundo vocacional. La primera instancia evaluadora es el propio sujeto, que valora lo que ha hecho con las opciones vocacionales que pretende; más tarde los biodatos pueden someterse a evaluaciones externas (selección de personal) respecto al mundo del trabajo.

El contenido de los biodatos lo podemos encuadrar en varias categorías: datos demográficos, académicos, familiares, de tiempo de ocio, relaciones sociales, etc. De todos ellos, en nuestro contexto, tal y como demuestran varias investigaciones previas (Rivas et al., 1998; Rocabert, 1995; Gimeno, 2004), son los biodatos académicos los que parecen mostrar una mayor relación con los intereses y las elecciones vocacionales de los estudiantes. 
Por ello el contar con los Biodatos académicos, y en concreto con la tasa educativa como variable de entrada es obligado en el estudio de la conducta vocacional. La investigación en nuestro país desvela que este indicador, respecto a la ocupación de llegada adulta, explica más que la tasa educativa y la ocupación de los padres (Castaño, 1983). El nivel educativo que tiene el individuo, en sus vertientes de rendimiento académico y experiencias educativas, es un codeterminante de primer orden (como por ejemplo el punto de corte establecido para el ingreso en determinadas titulaciones universitarias) para el acceso a los diferentes estratos del mundo ocupacional. La preparación escolar suele ser uno de los requisitos de entrada que contempla la diferenciación ocupacional, que en un escalonamiento formativo va de "Sin estudios" a "Doctor".

Otro indicador de los biodatos académicos hace referencia a los Itinerarios formativos que reflejan la formación escolar individual lograda a través de la opcionalidad de las asignaturas seguidas en los currícula escolares, tanto en secundaria como en otros niveles de enseñanza. En concreto, sistemas con escasa o nula optatividad restringen la diversidad en la formación y casi el desarrollo vocacional.

Por otra parte, el Rendimiento escolar actúa sobre el desarrollo vocacional futuro aunque no haya sido tenido en cuenta por el sujeto en periodos anteriores. Se cifra en el rendimiento escolar la equivalencia con el dominio o preparación instrumental entre los distintos niveles del sistema educativo, de hecho la mayoría de sistemas educativos toman el criterio rendimiento como filtro entre los diferentes niveles educativos, con informes y resultados de investigación de diferente signo. Así, aunque es conocida la escasa correlación entre el rendimiento en secundaria y el obtenido en pruebas de acceso con el rendimiento universitario (los resultados más aparentes no llegan a correlacionar .40), esa puntuación es la clave o representa la posibilidad de realizar determinados planes vocacionales, sin más justificación que la selectora.

Este tipo de biodatos académicos los hemos tenido en cuenta en aquellas investigaciones dirigidas a estudiar la conducta vocacional de estudiantes de secundaria, llegando a formar parte de las diferentes herramientas que para procesos de asesoramiento vocacional hemos venido elaborando desde hace algún tiempo (Rivas et al., 1998; Rivas, Rocabert, López, 2003). Dada su importancia a la hora de predecir elecciones vocacionales en la enseñanza secundaria, dentro del objetivo principal de la investigación referida a la 
conducta vocacional universitaria, nos planteamos la necesidad de elaborar un instrumento que nos permitiera analizar este codeterminante de la conducta vocacional universitaria. En este sentido, en primer lugar, se hacía preciso analizar y explorar qué tipo de biodatos, en la conducta vocacional universitaria, pueden estar condicionando la conducta vocacional de estudiantes y hasta qué punto se relacionan con otras variables que configuran esa conducta. Fundamentalmente nos interesaba constatar los biodatos de tipo académicos, que aportaran información sobre las vivencias, hechos y conductas del estudiante en relación a su desarrollo vocacional para, posteriormente, comprobar la adecuación o eficacia de las mismas a la hora de planificar y explorar sus opciones de especialización y preparación de su profesionalidad.

Por ello, además de los puros datos demográficos, nos interesaba analizar el grado de satisfacción de los estudiantes universitarios con sus estudios así como el tipo de actuaciones que un universitario ya ha realizado referidas todas ellas a procesos de toma de decisiones (elección de optativas, Practicum, etc.). De especial relevancia para el objetivo de nuestra investigación era el tener en cuenta el grado de decisión y clarificación de las decisiones en el ámbito profesional (salida profesional, especialización), así como las posibles dificultades encontradas para llevarlas a cabo. Por tanto, en nuestra propuesta se definen los Biodatos Universitarios a través de cinco categorías de variables: Motivación e Interés, Toma de Decisiones Académicas o Preprofesionales, Grado de Decisión, Experiencia Laboral y Dificultades percibidas en el Proceso de Toma de Decisiones.

\section{Método}

\section{Participantes}

En este estudio participaron un total de 2169 estudiantes que cursaban estudios universitarios en las doce universidades colaboradoras con el proyecto de investigación citado. El $34 \%$ de los sujetos eran varones y el $66 \%$ mujeres. La edad media fue de 23,05 años y la desviación típica de 3,18 . Todos los estudiantes participaron de forma voluntaria en la investigación, además de que cumplían el siguiente criterio de selección: "tener superado el $80 \%$ de los créditos de su titulación (equivalentes al penúltimo y último año de la carrera)”.

\section{Elaboración y descripción del instrumento}

Durante el proceso de elaboración del "Cuestionario de Biodatos Universitarios" se han considerado, en todo momento, las aportaciones correspondientes a: 1) un componente 
cualitativo, relativo al criterio personal de expertos en la materia que tratan de sacar a la luz las vivencias universitarias que caracterizan el desarrollo vocacional eficiente; y 2) un componente cuantitativo, referido a los resultados de diferentes estudios previos que permiten evaluar la relación de esos biodatos con un desarrollo vocacional eficaz de estudiantes universitarios.

Teniendo en cuenta estas consideraciones se elaboró el Cuestionario Biográfico Universitario, el CBU (Rocabert, 2005), que analiza la historia académica y los planes de futuro profesional del estudiante (Ver Anexo 1). El Cuestionario está compuesto por 9 indicadores que abarcan diferentes biodatos académicos relacionados con las experiencias vividas y conductas desplegadas por el estudiante universitario en relación a su planificación vocacional, permitiendo analizar el desarrollo vocacional del estudiante.

Se trata de un cuestionario informe que solicita al sujeto que seleccione aquella opción que mejor defina su situación en cada indicador, pudiendo, en algunas de ellas, escoger más de una alternativa o incluso elaborar alguna respuesta propia. Los biodatos que contempla el cuestionario se pueden agrupar en los siguientes apartados:

- Datos referidos a la Motivación e Interés que se tenía y se tiene en la actualidad por la opción universitaria escogida o trayectoria evolutiva del interés por lo que cursa, orden de elección (cuestiones 1, 2 y 3).

- Datos referidos a la Toma de Decisiones Académicas o Preprofesionales (Optativas, Practicum) (cuestiones 4 y 5).

- Datos referidos al Grado de Decisión sobre especialidad o salida profesional (cuestiones 6 y 8 ).

- Datos referidos a la Experiencia Laboral pasada o presente (cuestión 7).

- Datos referidos a las Dificultades percibidas en el Proceso de Toma de Decisiones preprofesionales (dificultades personales o de información académica-profesional) (cuestión 9).

Cada respuesta al cuestionario tiene asignado un valor, en función del grado en que contribuye o se relaciona con el desarrollo de carrera del estudiante (madurez vocacional, desarrollo de la identidad vocacional y conducta exploratoria). Las respuesta a cada una de las preguntas del cuestionario alcanzan una valoración entre 0 a 3 (excepto para las preguntas 8 y 9 que se limita a 2 puntos) siendo la máxima puntuación total posible de 26. 


\section{Procedimiento}

El Cuestionario de Biodatos Universitarios (CBU), como uno de los apartados del Protocolo 2005 dirigido a analizar la conducta vocacional universitaria, fue aplicado a los 2169 estudiantes de la muestra durante los meses de febrero a marzo de 2005. La aplicación se llevó a cabo en las distintas universidades participantes en el proyecto de investigación, de manera que los investigadores dirigían y coordinaban las condiciones de la aplicación en sus respectivas universidades.

Dicha aplicación pudo realizarse gracias a la participación de los "colaboradores de investigación", estudiantes universitarios, que participaban voluntariamente en seminarios de formación que les capacitaban para aplicar el protocolo 2005. Posteriormente procedían a la aplicación individualizada en términos de entrevista con los estudiantes universitarios que configuraban la muestra objeto de estudio.

Previamente a dicha aplicación y coincidiendo con el proceso de elaboración del Protocolo 2005, tuvo lugar una reunión entre los investigadores de las distintas universidades en la que se acordaron y clarificaron las directrices a seguir para organizar los seminarios de formación dirigidos a los colaboradores, así como las condiciones de la aplicación en general. Se movilizaron un total de 367 colaboradores de investigación que permitieron obtener 2169 protocolos válidos para el análisis de la conducta vocacional universitaria. El tratamiento de la información se llevó a cabo mediante el paquete estadístico SPSS (Statistical Package for Social Sciences) para Windows, versión 11.5.

En el estudio que nos ocupa, centrado en el Cuestionario de Biodatos Universitarios, se han realizado diferentes análisis descriptivos: Frecuencias y Porcentajes de elección de las diferentes opciones del cuestionario; Medias y Desviaciones Típicas, de las puntuaciones alcanzadas en las nueve cuestiones. También se ha calculado la homogeneidad interna del Cuestionario realizándose una correlación entre las nueve cuestiones y la puntuación total (P Biodatos) para analizar la posible relación existente entre ellas. Por último se han calculado, a partir de la puntuación total obtenida por los estudiantes en el Cuestionario (CBU), los puntos de corte que permiten segmentar a la población en grupos diferenciados de Desarrollo de Carrera Eficaz y que actuarán como variable criterio en el análisis de los diferentes ámbitos de la conducta vocacional de la investigación. 


\section{Resultados}

En cuanto a los análisis de frecuencias y porcentajes de elección, en la "Cuestión 1: Opción de Carrera" se analiza el orden de preferencia que manifestó el estudiante al entrar en la Universidad sobre los estudios que actualmente se encuentra realizando. Si observamos la tabla comprobamos que casi el 90\% (el 89,4\%) de la muestra afirma que los estudios que está cursando se encontraba entre la Opción Primera (el 70,9\%) y/o Segunda (18,6\%) de preferencia. Estos resultados vuelven a confirmar como la mayor parte de los estudiantes de secundaria pueden optar por su primera opción, aunque también es cierto que cerca del $20 \%$ se tiene que conformar con la segunda opción que poseían.

Tabla 1.- Frecuencias y porcentajes de Opción de la Carrera para la muestra total.

\begin{tabular}{|l|c|c|}
\hline Opción de carrera & Frecuencia & Porcentaje \\
\hline Primera & 1535 & $70,9 \%$ \\
Segunda & 402 & $18,6 \%$ \\
Tercera & 103 & $4,8 \%$ \\
Cuarta o más & 65 & $3,0 \%$ \\
Otras vías de acceso & 59 & $2,7 \%$ \\
Total & 2164 & $100,0 \%$ \\
\hline
\end{tabular}

La "Cuestión 2: Trayectoria Evolutiva del Interés por los Estudios" refleja el periodo de edad a partir del cual el universitario comienza a mostrar interés por sus estudios y permite por tanto analizar la trayectoria evolutiva de los intereses. Los porcentajes nos permiten confirmar, una vez más, que la secundaria postobligatoria es el periodo en el que parecen cristalizarse los intereses. Para un $62,6 \%$ de los universitarios, el interés por lo que cursan estaba ya presente a partir de los 17-18 años, mientras que tan sólo para un 11,8\% surgió en el periodo más temprano a los 12-14 años.

Tabla 2.- Frecuencias y porcentajes de la Evolución del Interés para la muestra total.

\begin{tabular}{|l|c|c|c|c|c|c|}
\cline { 2 - 7 } \multicolumn{1}{c|}{} & \multicolumn{2}{c|}{ Elegida } & \multicolumn{2}{c|}{ No Elegida } & \multicolumn{2}{c|}{ Total } \\
\cline { 2 - 7 } \multicolumn{1}{c|}{} & Frecuencia & Porcentaje & Frecuencia & Porcentaje & Frecuencia & Porcentaje \\
\hline Periodo 12-14 & 256 & $11,8 \%$ & 1911 & $88,2 \%$ & 2167 & $100,0 \%$ \\
Periodo 15-16 & 469 & $21,6 \%$ & 1698 & $78,4 \%$ & 2167 & $100,0 \%$ \\
Periodo 17-18 & 1356 & $62,6 \%$ & 811 & $37,4 \%$ & 2167 & $100,0 \%$ \\
Periodo 19 o más & 703 & $32,5 \%$ & 1462 & $67,5 \%$ & 2165 & $100,0 \%$ \\
\hline
\end{tabular}


La "Cuestión 3: Grado de Satisfacción con la Carrera" indaga sobre el grado de satisfacción con la opción universitaria que se está cursando. En trabajos anteriores esta cuestión permitió, como variable criterio, discriminar de forma muy adecuada a los mejores y peores estudiantes universitarios en relación a otros indicadores de la conducta vocacional, quizás como reflejo del carácter motivador que la satisfacción suele conllevar. Los resultados nos muestran que más de la mitad de la muestra se encuentra Muy satisfecha con lo que está estudiando (51,6\%). Si añadimos a estos sujetos los que se encuentran Bastante satisfechos (el $36,5 \%$ ), podemos concluir que alrededor del $85 \%$ de la muestra posee una alta satisfacción con lo que se está cursando, mientras que sólo un $2,8 \%$ se encuentra insatisfecho.

Tabla 3.- Frecuencias y porcentajes del Grado de Satisfacción con la Carrera para la muestra total

\begin{tabular}{|l|c|c|}
\hline Satisfacción en la elección & Frecuencia & Porcentaje \\
\hline Insatisfecho & 60 & $2,8 \%$ \\
Intermedio & 195 & $9,0 \%$ \\
Intermedio & 788 & $36,5 \%$ \\
Muy Satisfecho & 1113 & $51,6 \%$ \\
Total & 2156 & $100,0 \%$ \\
\hline
\end{tabular}

La "Cuestión 4: Elección de Asignaturas Optativas" analiza los criterios seguidos por el estudiante a la hora de escoger las asignaturas optativas de su Plan de Estudios, pudiéndose reflejar mas de una opción. El 59,1\% de la muestra escoge las optativas por el interés en sus contenidos, siendo por tanto los gustos y las preferencias el mayor motivador de la elección, mientras que un 52,5\% las escogen, basándose su relación con el trabajo o especialidad futura. Pero también es preciso destacar que es muy alto el porcentaje de estudiantes que afirman elegirlas en función del Horario $(43,4 \%)$ o por la Facilidad de la materia $(31,0 \%)$.

Tabla 4.- Frecuencias y porcentajes de Elección de las Asignaturas Optativas para la muestra total.

\begin{tabular}{|l|c|c|c|c|c|c|}
\cline { 2 - 6 } \multicolumn{1}{c|}{} & \multicolumn{2}{c|}{ Elegida } & \multicolumn{2}{c|}{ No Elegida } & \multicolumn{2}{c|}{ Total } \\
\cline { 2 - 7 } & Frecuencia & Porcentaje & Frecuencia & Porcentaje & Frecuencia & Porcentaje \\
\hline Relación con la especialidad & 1138 & $52,5 \%$ & 1031 & $47,5 \%$ & 2169 & $100,0 \%$ \\
o trabajo futuro & 942 & $43,4 \%$ & 1227 & $56,5 \%$ & 2169 & $100,0 \%$ \\
Horario & 673 & $31,0 \%$ & 1495 & $69,0 \%$ & 2169 & $100,0 \%$ \\
Elecilidad de la materia & 223 & $10,3 \%$ & 1946 & $89,7 \%$ & 2169 & $100,0 \%$ \\
Profesor/a & 401 & $18,5 \%$ & 1768 & $81,5 \%$ & 2169 & $100,0 \%$ \\
Interés de los contenidos & 1282 & $59,1 \%$ & 887 & $40,9 \%$ & 2169 & $100,0 \%$ \\
No existe optatividad & 142 & $6,5 \%$ & 227 & $93,5 \%$ & 2169 & $100,0 \%$ \\
\hline
\end{tabular}


Al igual que en la pregunta anterior, en la "Cuestión 5: Elección del Practicum" se analizan los criterios de elección pero en este caso referidos al Practicum. Los datos reflejan que un $44,3 \%$ de los universitarios afirma seleccionarlo en función de las posibilidades de Salidas Profesionales futuras, y un 44,2\% en función de la Especialidad que quiere realizar. Es de destacar el bajo porcentaje de estudiantes que "no lo tienen claro" o que "no han pensado en ello", representando un porcentaje cercano al 5\%. Tan sólo un 5,6\% de los universitarios afirma no haber podido escoger libremente su Practicum.

Tabla 5.- Frecuencias y porcentajes de la Elección del Practicum para la muestra total

\begin{tabular}{|c|c|c|c|c|c|c|}
\hline & \multicolumn{2}{|c|}{ Elegida } & \multicolumn{2}{|c|}{ No Elegida } & \multicolumn{2}{|c|}{ Total } \\
\hline & Frecuencia & Porcentaje & Frecuencia & Porcentaje & Frecuencia & Porcentaje \\
\hline $\begin{array}{l}\text { Horario y proximidad a } \\
\text { casa }\end{array}$ & 394 & $18,2 \%$ & 1774 & $81,8 \%$ & 2168 & $100,0 \%$ \\
\hline $\begin{array}{l}\text { Interés por las tareas que } \\
\text { allí se realizan }\end{array}$ & 770 & $35,5 \%$ & 1399 & $64,5 \%$ & 2169 & $100,0 \%$ \\
\hline $\begin{array}{l}\text { Buenas referencias de la } \\
\text { plaza o empresa }\end{array}$ & 470 & $21,7 \%$ & 1699 & $78,3 \%$ & 2169 & $100,0 \%$ \\
\hline $\begin{array}{l}\text { No tuviste posibilidad de } \\
\text { elección }\end{array}$ & 122 & $5,6 \%$ & 2043 & $94,4 \%$ & 2165 & $100,0 \%$ \\
\hline $\begin{array}{l}\text { Orientación de tu tutor/a } \\
\text { de prácticas }\end{array}$ & 236 & $10,9 \%$ & 1933 & $89,1 \%$ & 2169 & $100,0 \%$ \\
\hline No existen prácticas & 217 & $10,0 \%$ & 1947 & $90,0 \%$ & 2164 & $100,0 \%$ \\
\hline $\begin{array}{l}\text { Posibilidades de futuras } \\
\text { salidas profesionales }\end{array}$ & 961 & $44,3 \%$ & 1208 & $55,7 \%$ & 2169 & $100,0 \%$ \\
\hline No he pensado en ello & 119 & $5,5 \%$ & 2049 & $94,5 \%$ & 2168 & $100,0 \%$ \\
\hline $\begin{array}{l}\text { Especialidad a la que } \\
\text { quiero dedicarme }\end{array}$ & 958 & $44,2 \%$ & 1211 & $55,8 \%$ & 2169 & $100,0 \%$ \\
\hline No tengo claro qué elegir & 100 & $4,6 \%$ & 2068 & $95,4 \%$ & 2168 & $100,0 \%$ \\
\hline
\end{tabular}

La "Cuestión 6: Decisión sobre la Especialidad que se desea seguir" facilita información sobre la especialización. Permite tal y como está formulada no sólo recabar información sobre el grado de decisión de los universitarios en relación a su especialización, sino también concretar la especialización o especializaciones posibles (aunque aquí no vamos a comentar estos datos). Por último, recaba información sobre la necesidad de ayuda para adoptar este tipo de decisión, en el caso de no tenerlo claro. Esta información es de mucha utilidad para los servicios de asesoramiento vocacionales universitarios a la hora de planificar sus intervenciones. Los resultados de nuestro estudio informan que el 41,8\% tiene Decidido la especialidad profesional, un 26,6\% No lo tiene decidido y un 9,0\% Necesitaría ayuda. 
Tabla 6.- Frecuencias y porcentajes de Especialidad Profesional para la muestra total.

\begin{tabular}{|l|c|c|c|c|}
\cline { 2 - 5 } \multicolumn{1}{c|}{} & \multicolumn{2}{c|}{ Elegida } & \multicolumn{2}{c|}{ No Elegida } \\
\cline { 2 - 5 } \multicolumn{1}{c|}{} & Frecuencia & Porcentaje & Frecuencia & Porcentaje \\
\hline Tengo muy claro lo que quiero hacer & 905 & $41,8 \%$ & 1262 & $58,2 \%$ \\
Dudo entre dos especialidades & 301 & $13,9 \%$ & 1868 & $86,1 \%$ \\
Dudo entre tres especialidades & 189 & $8,7 \%$ & 1980 & $91,3 \%$ \\
No tengo decidido nada & 576 & $26,6 \%$ & 1591 & $73,4 \%$ \\
Necesitaría ayuda & 196 & $9,0 \%$ & 1973 & $91,0 \%$ \\
\hline
\end{tabular}

La "Cuestión 7: Experiencia Laboral" analiza la experiencia laboral actual o pasada del estudiante, así como en el caso de haberla tenido, su relación o no con los estudios que se está cursando. Los resultados nos informan que cerca de un $40 \%$ de los estudiantes (el 36,5\%) afirma haber tenido experiencias laborales aunque ahora no trabajen, mientras que cerca de un $29,0 \%$ no ha trabajado nunca. Sólo un $13,7 \%$ trabaja en algo relacionado con sus estudios.

Tabla 7.- Frecuencias y porcentajes de Experiencia Laboral para la muestra total.

\begin{tabular}{|l|c|c|c|c|c|c|}
\cline { 2 - 6 } \multicolumn{1}{c|}{} & \multicolumn{2}{c|}{ Elegida } & \multicolumn{2}{c|}{ No Elegida } & \multicolumn{2}{c|}{ Total } \\
\cline { 2 - 7 } \multicolumn{1}{l|}{} & Frecuencia & Porcentaje & Frecuencia & Porcentaje & Frecuencia & Porcentaje \\
\hline $\begin{array}{l}\text { No trabajo, pero he tenido } \\
\text { experiencias laborales } \\
\begin{array}{l}\text { Trabajo en algo relacionado } \\
\text { con lo que estudio }\end{array}\end{array}$ & 791 & $36,5 \%$ & 1378 & $63,5 \%$ & 2169 & $100,0 \%$ \\
$\begin{array}{l}\text { Trabajo, pero no relacionado } \\
\text { con lo que estudio }\end{array}$ & 439 & $13,7 \%$ & 1871 & $86,3 \%$ & 2169 & $100,0 \%$ \\
No he trabajado nunca & 630 & $29,0 \%$ & 1728 & $79,7 \%$ & 2167 & $100,0 \%$ \\
\hline
\end{tabular}

La "Cuestión 8: Decisión al Finalizar sus Estudios" analiza si se ha adoptado ya una decisión sobre lo que tiene previsto hacer al finalizar los actuales estudios, permitiendo rastrear las diferentes posibilidades presentes o pasadas, así como la relación con lo que se está estudiando. Los resultados nos muestran que al finalizar los estudios la mayoría, el 48,8\%, desean Ejercer la profesión, un 34,6\% Realizar un Máster y un 30,4\% piensan Opositar. Destaca el poco porcentaje de estudiantes, tan sólo un $1,7 \%$, que todavía No lo ha pensado, aunque un $13,7 \%$ de los estudiantes, todavía No lo han decidido. 
Tabla 8.- Frecuencias y porcentajes de la Decisión a adoptar cuando finalicen los estudios para la muestra total (se han eliminado las especificaciones de carreras).

\begin{tabular}{|l|c|c|c|c|}
\cline { 2 - 5 } \multicolumn{1}{c|}{} & \multicolumn{2}{c|}{ Elegida } & \multicolumn{2}{c|}{ No Elegida } \\
\cline { 2 - 5 } \multicolumn{1}{c|}{} & Frecuencia & Porcentaje & Frecuencia & Porcentaje \\
\hline Ejercer la profesión & 1059 & $48,8 \%$ & 1110 & $51,2 \%$ \\
Hacer un máster & 751 & $34,6 \%$ & 1418 & $65,4 \%$ \\
Hacer el doctorado & 233 & $10,7 \%$ & 1936 & $89,3 \%$ \\
Solicitar beca & 202 & $9,3 \%$ & 1967 & $90,7 \%$ \\
Hacer oposiciones & 659 & $30,4 \%$ & 1510 & $69,6 \%$ \\
Buscar un trabajo cualquiera & 253 & $11,7 \%$ & 1915 & $88,3 \%$ \\
No lo he decidido & 297 & $13,7 \%$ & 1872 & $86,3 \%$ \\
Otra carrera & 348 & $16,1 \%$ & 1817 & $83,9 \%$ \\
\hline
\end{tabular}

La “Cuestión 9: Dificultades y /o Problemas en la Transición al mundo laboral” indaga los diferentes problemas o dificultades que pueden surgir ante la decisión a adoptar en el momento de la transición al mundo laboral. Los resultados indican que a la hora de escoger una especialidad, un 33\% No sabe cual es la que más le conviene, un 27,8\% afirma que No tiene dificultades personales para decidirse pero un $26,7 \%$ cree que le Falta información sobre las características de las especialidades. Por otra parte, sólo un 12,5\% tiene información académico-profesional.

Tabla 9.- Frecuencias y porcentajes de Dificultades o Problemas para decidir especialidad o salida profesional, para la muestra total.

\begin{tabular}{|c|c|c|c|c|c|c|}
\hline & \multicolumn{2}{|c|}{ Elegida } & \multicolumn{2}{|c|}{ No Elegida } & \multicolumn{2}{|c|}{ Total } \\
\hline & Frecuencia & Porcentaje & Frecuencia & Porcentaje & Frecuencia & Porcentaje \\
\hline $\begin{array}{l}\text { Ninguna opción me satisface } \\
\text { plenamente }\end{array}$ & 175 & $8,1 \%$ & 1993 & $91,9 \%$ & 2168 & $100,0 \%$ \\
\hline $\begin{array}{l}\text { No tengo claro la opción que más } \\
\text { me conviene }\end{array}$ & 715 & $33,0 \%$ & 1454 & $67,0 \%$ & 2169 & $100,0 \%$ \\
\hline $\begin{array}{l}\text { Me falta información fiable sobre } \\
\text { mí mismo }\end{array}$ & 217 & $10,0 \%$ & 1952 & $90,0 \%$ & 2169 & $100,0 \%$ \\
\hline $\begin{array}{l}\text { Que mis preferencias } \\
\text { profesionales cambien en el futuro }\end{array}$ & 312 & $14,4 \%$ & 1854 & $85,6 \%$ & 2166 & $100,0 \%$ \\
\hline $\begin{array}{l}\text { Desconozco qué es importante } \\
\text { tener en cuenta para decidirme }\end{array}$ & 244 & $11,2 \%$ & 1925 & $88,8 \%$ & 2169 & $100,0 \%$ \\
\hline $\begin{array}{l}\text { No tengo contactos que me ayuden } \\
\text { a conseguir el trabajo que quiero }\end{array}$ & 365 & $16,9 \%$ & 1798 & $83,1 \%$ & 2163 & $100,0 \%$ \\
\hline $\begin{array}{l}\text { Personas importantes para mí, no } \\
\text { apoyan mis planes }\end{array}$ & 36 & $1,7 \%$ & 2133 & $98,3 \%$ & 2169 & $100,0 \%$ \\
\hline $\begin{array}{l}\text { Me falta información sobre las } \\
\text { características de la especialidad }\end{array}$ & 579 & $26,7 \%$ & 1586 & $73,3 \%$ & 2165 & $100,0 \%$ \\
\hline $\begin{array}{l}\text { Tengo la información académico- } \\
\text { profesional que me hace falta }\end{array}$ & 604 & $27,8 \%$ & 1565 & $72,2 \%$ & 2169 & $100,0 \%$ \\
\hline & 272 & $12,5 \%$ & 1897 & $87,5 \%$ & 2169 & $100,0 \%$ \\
\hline
\end{tabular}


Respecto a los análisis descriptivos, las medias más altas se obtienen en la Cuestión Primera (Opción universitaria) con una media cercana a 3, por lo que podemos concluir que los estudiantes universitarios parecen estar realizando aquellos estudios universitarios que al finalizar la secundaria se encontraban entre la primera y la segunda opción, aunque más cerca de la primera que de la segunda. La segunda media mas alta la obtiene la Cuestión 6 que se refiere a la decisión sobre la especialización, de manera que, según indican estos datos, los estudiantes parecen tener decidida la especialidad o tan sólo estar dudando entre dos de ellas. Por último con una media de 2,30 aparece la Cuestión 5 que hace referencia a la elección del Practicum, para cuya elección parecen manejar criterios adecuados.

En el polo opuesto se sitúa la Cuestión 9 que es en la que se observa la media mas baja cercana a 1, sin embargo, es importante matizar que en esta pregunta sólo se puede alcanzar una valoración máxima de 2. En todo caso una media cercana a 1 supone que la mayoría de los estudiantes tienen alguna dificultad ya sea de información vocacional o de conocimiento de su conducta vocacional, reflejando además esta cuestión la más baja desviación típica. También cercana a una media de 1 se sitúan las Cuestiones 2 referida a los periodos de edad en los que se muestra interés por lo que se está cursando $(1,21)$ y la cuestión 4 , que analiza los criterios a la hora de escoger asignaturas optativas (con una media de 1,41).

Tabla 10.- Descriptivos de las cuestiones del Cuestionario de Biodatos Universitario (CBU).

\begin{tabular}{|l|c|c|c|c|c|}
\hline CUESTIÓN & $\mathbf{N}$ & $\begin{array}{c}\text { Valor } \\
\text { mínimo }\end{array}$ & $\begin{array}{c}\text { Valor } \\
\text { máximo }\end{array}$ & Media & $\begin{array}{c}\text { Desviación } \\
\text { típica }\end{array}$ \\
\hline 1. Opción de carrera & 1937 & 2 & 3 & 2,79 & 0,41 \\
\hline 2. Evolución del interés & 1726 & 1 & 3 & 1,21 & 0,53 \\
\hline 3. Satisfacción con los estudios & 2096 & 1 & 3 & 2,44 & 0,66 \\
\hline 4. Optativas & 1711 & 1 & 2 & 1,41 & 0,49 \\
\hline 5. Practicum & 958 & 1 & 3 & 2,30 & 0,74 \\
\hline 6. Decisión especialidad & 1375 & 1 & 3 & 2,51 & 0,73 \\
\hline 7. Experiencia laboral & 1521 & 1 & 3 & 1,67 & 0,78 \\
\hline 8. Decisión al finalizar & 1890 & 1 & 3 & 1,93 & 0,83 \\
\hline 9. Dificultades toma de decisión & 755 & 1 & 2 & 1,16 & 0,37 \\
\hline
\end{tabular}

Con el fin de ver las posibles relaciones entre las diferentes cuestiones del Cuestionario y la Puntuación Biodatos Total, se procedió a realizar un análisis correlacional para comprobar su homogeneidad interna (Tabla 11). Sólo la "Experiencia Laboral” (cuestión 7) no se correlaciona con ninguna otra variable, excepto con la Total. Por el contrario la Cuestión 3: la Satisfacción con lo que se está realizando, correlaciona con todas menos con dos, con la Cuestión 7: Experiencia laboral y la Cuestión 8: Decisión a adoptar cuando se 
finalicen los estudio. La Decisión al finalizar los estudios actuales, correlaciona significativamente con todas excepto con la cuestión 7: Experiencia Laboral, la cuestión 6: Especialización de la Carrera, la cuestión 9: Dificultades o Problemas a la hora de decidir especialidad o área de trabajo y la cuestión 1: Opción de Carrera.

Tabla 11.- Correlación entre las nueve cuestiones del CBU y la Puntuación de Biodatos Total (BIO).

\begin{tabular}{|c|c|c|c|c|c|c|c|c|c|c|c|}
\hline & & CUES1 & CUEST2 & CUES3 & CUES4 & CUES5 & CUES6 & CUES7 & CUES8 & CUEST9 & $\mathrm{BlO}$ \\
\hline \multirow[t]{3}{*}{ CUES1 } & Correlación de Pearson & 1 &, $110^{* *}$ &, $140^{* *}$ &, $064^{*}$ &, 048 &, $061^{*}$ &, 040 & ,019 &, 029 &, $237^{* *}$ \\
\hline & Sig. (bilateral) & , &, 000 &, 000 & ,011 & 153 & 032 & 142 &, 434 &, 450 &, 000 \\
\hline & $\mathrm{N}$ & 1937 & 1628 & 1878 & 1545 & 872 & 1240 & 1352 & 1698 & 679 & 1937 \\
\hline \multirow[t]{3}{*}{ CUEST2 } & Correlación de Pearson &, $110^{* *}$ & 1 & $078^{* *}$ & , 125** & ,036 &, 025 & ,052 &, $112^{* *}$ & ,007 &, $313^{+x}$ \\
\hline & Sig. (bilateral) &, 000 & , &, 001 &, 000 &, 312 &, 407 & ,076 &, 000 & 866 &, 000 \\
\hline & $\mathrm{N}$ & 1628 & 1726 & 1672 & 1390 & 779 & 1108 & 1161 & 1509 & 604 & 1726 \\
\hline \multirow[t]{3}{*}{ CUES3 } & Correlación de Pearson &, $140^{* *}$ &, $078^{* *}$ & 1 & $141^{* *}$ & $079^{*}$ &, $070^{* *}$ &, 011 &, $108^{+* x}$ & $099^{*+}$ &, $455^{*}$ \\
\hline & Sig. (bilateral) &, 000 &, 001 & , &, 000 & ,016 &, 010 &, 660 &, 000 & ,007 &, 000 \\
\hline & N & 1878 & 1672 & 2096 & 1663 & 932 & 1341 & 1470 & 1833 & 733 & 2096 \\
\hline \multirow[t]{3}{*}{ CUES4 } & Correlación de Pearson &, $064^{*}$ & $125^{* *}$ &, $141^{* *}$ & 1 & ,249* &, 041 & ,024 &, $118^{\text {t*x }}$ &, $136^{* *}$ &, $379^{*}$ \\
\hline & Sig. (bilateral) &, 011 &, 000 &, 000 & , &, 000 & , 165 & ,399 &, 000 & ,001 &, 000 \\
\hline & $\mathrm{N}$ & 1545 & 1390 & 1663 & 1711 & 825 & 1134 & 1220 & 1512 & 631 & 1711 \\
\hline \multirow[t]{3}{*}{ CUES5 } & Correlación de Pearson &, 048 & ,036 &, $079^{*}$ & $249^{* *}$ & 1 &,- 046 &,- 038 &, $110^{* *}$ & ,021 &, $314^{* *}$ \\
\hline & Sig. (bilateral) & ,153 &, 312 & 016 &, 000 & , & ,228 &, 317 &, 001 &, 693 &, 000 \\
\hline & $\mathrm{N}$ & 872 & 779 & 932 & 825 & 958 & 692 & 706 & 883 & 371 & 958 \\
\hline \multirow[t]{3}{*}{ CUES6 } & Correlación de Pearson &, $061^{*}$ &, 025 &, $070^{* *}$ &, 041 &,- 046 & 1 &, 040 &,- 035 & 057 &, $336^{* *}$ \\
\hline & Sig. (bilateral) & ,032 &, 407 &, 010 & 165 & ,228 & , & ,202 & ,210 & ,152 &, 000 \\
\hline & $\mathrm{N}$ & 1240 & 1108 & 1341 & 1134 & 692 & 1375 & 1036 & 1280 & 633 & 1375 \\
\hline \multirow[t]{3}{*}{ CUES7 } & Correlación de Pearson &, 040 & ,052 & ,011 & ,024 &,- 038 &, 040 & 1 &,- 024 &, 007 & $232 *$ \\
\hline & Sig. (bilateral) & ,142 & ,076 &, 660 & ,399 & ,317 & 202 & , & ,386 & 870 &, 000 \\
\hline & $\mathrm{N}$ & 1352 & 1161 & 1470 & 1220 & 706 & 1036 & 1521 & 1349 & 582 & 1521 \\
\hline \multirow[t]{3}{*}{ CUES8 } & Correlación de Pearson & ,019 &, $112^{* *}$ & $108^{* *}$ &, $118^{\star *}$ &, $110^{* *}$ &,- 035 &,- 024 & 1 &, $079^{*}$ & $347^{*+4}$ \\
\hline & Sig. (bilateral) &, 434 &, 000 &, 000 &, 000 &, 001 & ,210 &, 386 & & ,036 &, 000 \\
\hline & $\mathrm{N}$ & 1698 & 1509 & 1833 & 1512 & 883 & 1280 & 1349 & 1890 & 712 & 1890 \\
\hline \multirow[t]{3}{*}{ CUEST9 } & Correlación de Pearson &, 029 & ,007 & $099 *$ & $136^{* *}$ & ,021 & ,057 &, 007 & $079^{*}$ & 1 &, $271^{*+*}$ \\
\hline & Sig. (bilateral) &, 450 & 866 &, 007 &, 001 &, 693 & ,152 &, 870 & ,036 & , &, 000 \\
\hline & $\mathrm{N}$ & 679 & 604 & 733 & 631 & 371 & 633 & 582 & 712 & 755 & 755 \\
\hline \multirow[t]{3}{*}{$\mathrm{BIO}$} & Correlación de Pearson & $237^{7 *}$ &, $313^{* *}$ &, $455^{* *}$ &, $379^{* *}$ &, $314^{* *}$ &, $336^{* *}$ &, $232^{* *}$ &, $347^{7 *}$ &, $271^{* *}$ & 1 \\
\hline & Sig. (bilateral) &, 000 &, 000 &, 000 &, 000 &, 000 &, 000 &, 000 &, 000 &, 000 & , \\
\hline & $\mathrm{N}$ & 1937 & 1726 & 2096 & 1711 & 958 & 1375 & 1521 & 1890 & 755 & 2169 \\
\hline
\end{tabular}

Los resultados muestran como todas las cuestiones correlacionan con la Puntuación Total de Biodatos siendo a su vez todas ellas significativas al nivel de 0,01. La Cuestión 3: Satisfacción con lo que se está realizando es la que alcanza una correlación mas alta (.455); mientras que la Cuestión 7: Experiencia Laboral es la que menos correlación obtiene (.232), aunque también es significativa. El Cuadro 1 resume estos resultados. 


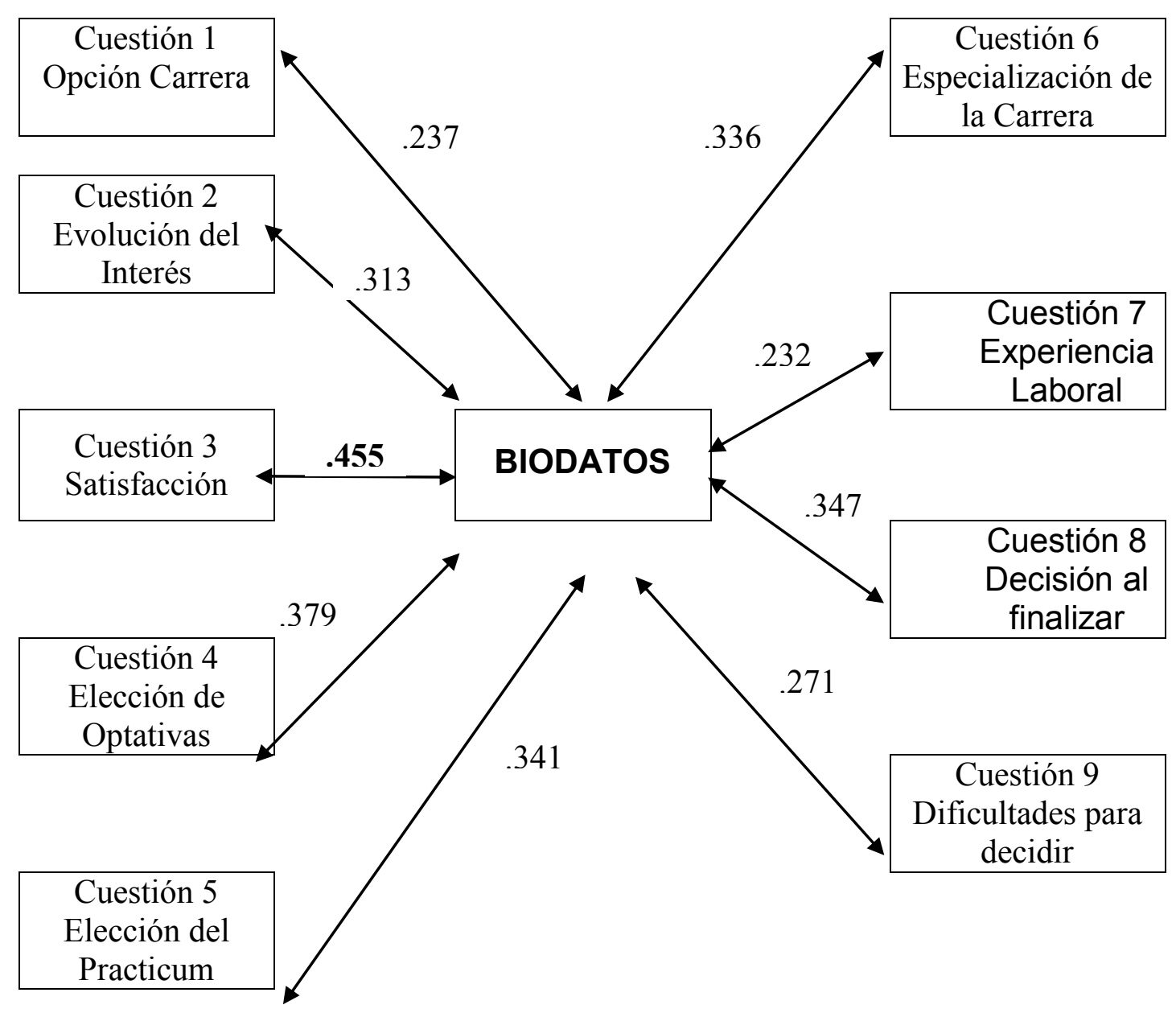

Cuadro 1.- Correlaciones de las cuestiones de Biodatos y la Puntuación de Biodatos Total.

Por último, en función de la Puntuación Total de BIODATOS se tomó la decisión de segmentar a la población tomando como referencia el $25 \%$ superior e inferior asimilándose a los cuartiles extremos poblacionales $\left(\mathrm{Q}_{1} \mathrm{y} \mathrm{Q}_{3}\right)$. De esta manera surgen tres subgrupos: El subgrupo 3 engloba a los universitarios con un Desarrollo de Carrera Eficaz; mientras que el subgrupo 1 recoge a aquellos universitarios con un Desarrollo de carrera No eficaz; situándose en el Subgrupo 2 el resto de estudiantes.

En la Tabla 12 se puede comprobar como cerca de un $26 \%$ de los estudiantes poseen un desarrollo de carrera eficaz, mientras que cercano al $30 \%$ los calificaríamos con un desarrollo de carrera no eficaz. 
Tabla 12.- Frecuencias y porcentajes de los Subgrupos de Biodatos (Desarrollo de Carrera)

\begin{tabular}{|c|c|c|}
\hline $\begin{array}{c}\text { SUBGRUPOS DE BIODATOS } \\
\text { (Desarrollo de Carrera) }\end{array}$ & Frecuencia & Porcentaje \\
\hline $\begin{array}{c}\mathbf{1} \\
\text { Desarrollo de Carrera No Eficaz }\end{array}$ & 624 & $28,8 \%$ \\
\hline $\begin{array}{c}\mathbf{2} \\
\text { Desarrollo Intermedio }\end{array}$ & 983 & $45,3 \%$ \\
\hline $\mathbf{3}$ & 562 & 25,95 \\
\hline Desarrollo de Carrera Eficaz & 2169 & $100,0 \%$ \\
\hline TOTAL & \multicolumn{2}{|}{} \\
\hline
\end{tabular}

\section{Discusión y conclusiones}

El propósito de analizar la naturaleza de las experiencias vividas por parte de estudiantes universitarios y su planteamiento vocacional a lo largo de su tránsito por la formación universitaria, tal y como hemos venido realizando con estudiantes de enseñanza secundaria, nos llevó a elaborar el Cuestionario de Biodatos Universitarios (CBU) que se ciñe al análisis de los biodatos académicos y que se ha comportado como un buen indicador del desarrollo vocacional de una muestra de 2169 estudiantes que cursan los últimos cursos de sus estudios en doce universidades españolas.

Los análisis de homogeneidad del cuestionario muestran como sus nueve cuestiones que abarcan diferentes ámbitos (Motivación e Interés, Toma de Decisiones académicas y preprofesionales, Grado de Decisión, Experiencia Laboral y Dificultades en el Proceso de Toma de Decisiones) correlacionan significativamente $(0,01)$ con la Puntuación Total de Biodatos. La satisfacción por lo que se cursa muestra las mayores correlaciones con la puntuación total mientras que la experiencia laboral es la que más se aleja de ella (ver Cuadro $\mathrm{n}^{\mathrm{o}} 1$ ). Asimismo hay que destacar que, a pesar de las relaciones existentes entre las diferentes cuestiones, las correlaciones no son demasiado altas como para dar información redundante.

Los análisis descriptivos del CBU nos dibuja una muestra universitaria altamente satisfecha con lo que cursa, siendo muy minoritario, tan sólo un $2,8 \%$, el porcentaje de estudiantes que se muestra insatisfecho. Este grado de satisfacción era esperable en los estudiantes que escogieron la opción universitaria más preferida (el 70,8\%) pero no tanto para cerca del $20 \%$ de sujetos que en su momento de la elección de estudios universitarios escogieron su segunda opción en preferencia. A pesar de ello, los estudiantes parecen 
adaptarse a los estudios definitivamente escogidos de una manera satisfactoria ya que el $85 \%$ de los estudiantes relata que está muy satisfecho o al menos satisfecho con lo que estudia.

Respecto a la evolución de sus intereses profesionales los resultados parecen confirmar que en la mayoría de los universitarios el interés por los estudios cursados estaba ya presente a partir de los 17-18 años, siendo éste, el interés, una de las razones que les lleva a escoger las asignaturas optativas, junto con el grado de relación existente con la futura opción profesional. El universitario mayoritariamente (casi el $60 \%$ de los estudiantes) utiliza, a la hora de tomar decisiones, criterios maduros y racionales vocacionalmente, pero sigue existiendo un porcentaje demasiado alto de estudiantes que afirma elegir asignaturas por su horario y/o la facilidad de la materia (el $43 \%$ y $31 \%$, respectivamente). Si nos ceñimos a la elección del Practicum la mayoría lo escoge o piensa escogerlo por criterios racionales como la relación con el trabajo, o la especialidad, siendo muy pocos los estudiantes que no lo tienen claro todavía, o los que no lo han escogido voluntariamente (alrededor de un 5,5\% de los estudiantes en ambos casos).

Cuando la decisión afecta a la especialidad o salida profesional los resultados no son tan positivos. Lo más destacable de la elección de la especialidad profesional es su alto porcentaje de indecisos, más del $30 \%$ de la muestra no la tiene todavía clarificada, expresando un $9 \%$ de estudiantes la necesidad de recibir asesoramiento para tomar este tipo de decisión. Si tenemos en cuenta que tan sólo un $12,5 \%$ de los universitarios afirma tener la información académico-profesional necesaria, y que un $11,2 \%$ de universitarios desconoce qué es importante tener en cuenta para adoptar este tipo de decisiones, se hace evidente la necesidad de cubrir la falta de información y de poner en marcha servicios de asesoramiento vocacional especializado en este tramo de la formación, pues la mayoría de los estudiantes tienen alguna dificultad, ya sea de información vocacional o de conocimiento de su propia conducta vocacional.

Pero más allá de la utilidad que este trabajo tiene a la hora de describir los biodatos universitarios, tan escasamente relatados en nuestro contexto, lo más relevante, a nuestro parecer, es la obtención, a través del CBU, de una puntuación total de biodatos. En este sentido, a pesar de la variedad de aspectos teóricos que se incluyen en los biodatos, hemos conseguido elaborar una herramienta que permite obtener por primera vez una puntuación global que a su vez se ha comportado adecuadamente a la hora de clasificar a los estudiantes 
en diferentes grupos de desarrollo de carrera desde el punto de vista de su grado de efícacia. El criterio utilizado a la hora de segmentar a la población en base a esa puntuación ha demostrado su bondad psicométrica permitiéndonos clasificar a los sujetos en tres subgrupos, según su grado de eficacia en el Desarrollo de Carrera. Los análisis en nuestro estudio clasifica a un $26 \%$ de los estudiantes con un Desarrollo de Carrera Eficaz, mientras que cercano al 30\% los sitúa con un Desarrollo de Carrera No Eficaz. Esta clasificación de los universitarios en función de los biodatos y su grado de desarrollo eficaz ha mostrado ser un indicador válido a la hora de predecir el adecuado desarrollo de los estudiantes en otros indicadores vocacionales: Identidad Vocacional (Pascual, 2006, Rivas et al, 2005), Personalidad Eficiente (Gómez, Rocabert, Descals, 2006; Rivas y Rocabert, 2005) y Estudio (Rivas y Rocabert, 2005).

Como prospectiva de investigación, pensamos que los resultados de este estudio deben completarse con otros trabajos en los que se aborden aspectos aquí obviados. Entre ellos podría resaltarse el análisis diferenciado de los biodatos en función del tipo de estudios que se cursan o Grupo Vocacional, del género de los estudiantes; así como aquellos que validen la utilidad del CBU como predictor de otros indicadores de la conducta vocacional: identidad, personalidad, estudio, cognición. 


\section{Referencias bibliográficas}

Castaño, C. (1983). Psicología y Orientación Vocacional. Madrid: Morata.

Farmer, H. (1985). Model of career and achievement motivation for women and men. Journal of Counseling Psychology, 32, 363-390.

Gimeno, M.J. (2004). Análisis de la problemática vocacional y de los biodatos ante la Toma de Decisiones. Tesis Doctoral. Universidad de Valencia.

Gómez, A.; Rocabert E. y Descals, A. (2006). "Elaboración de un cuestionario de personalidad eficiente para el análisis de la conducta vocacional universitaria" Revista Galego/Portuguesa de Psicoloxia e Educación, 11, 13, 399-411.

O’Neil, J. M.; Ohlde, C.; Tollefson, N.; Barke, C.; Piggott, T. y Watts, D. (1980). Factors, correlates and problem areas affecting career decision making of a cross sectional sample of students. Journal of Counseling Psychology, 6, 571-580.

Owens, S. (1983). Background, cap. 14 en Dunnette, Handbook of Industrial and Organizational Psychology, Wiley-Interscience, University of Minnesota.

Pascual, F. (2006). Conducta e Identidad Vocacional: Una aproximación Empírica. Tesis Doctoral. Universidad de Valencia.

Rivas, F.; Rocabert, E.; Ardit, I.; J.R. Martínez y J.M. Rius (1998). Carpeta para el profesional del SAAV: Manual del SAV-R y del SAVI-2000. Para uso profesional del Orientador. Servicio de Asesoramiento Vocacional y Educativos S.L.

Rivas, F.; Rocabert, E.; López, M.L. (2003). Sistemas de Autoayuda y Asesoramiento Vocacional (SAAV). Madrid: EOS.

Rivas, F. y Rocabert, E. (2005). Conducta vocacional y asesoramiento profesional de los estudiantes universitarios: Protocolo informático para la autoayuda preprofesional. Informe final del proyecto de investigación I + D BSO2001-3150, financiado por el Ministerio de Ciencia y Tecnología.

Rocabert, E. (1995). Historia personal. En F. Rivas (ed.), Manual de asesoramiento y orientación vocacional. Madrid: Sintesis.

Rocabert, E. (2005). Biodatos en F. Rivas y E. Rocabert, (2005). Conducta vocacional y asesoramiento profesional de los estudiantes universitarios: Protocolo informático para la autoayuda preprofesional. Informe final del proyecto de investigación $I+D$ BSO2001-3150, financiado por el Ministerio de Ciencia y Tecnología. 


\section{ANEXO 1: CUESTIONARIO DE BIODATOS UNIVERSITARIOS (CBU)}

Te presentamos una serie de cuestiones que hacen referencia a algunos datos sobre tu trayectoria académica. Responde seleccionando aquella opción u opciones que mejor reflejen tu caso.

1. Al finalizar la Selectividad u otras vías de acceso a la Universidad, la carrera que cursas AHORA fue la opción que elegiste en el lugar: (Señalar con $X$ )
$\square$ Primero
$\square$ Segundo
$\square$ Tercero
$\square$ Cuarto o más
Otras vías de acceso

2. ¿Durante qué periodos te interesaron los estudios que estás cursando? (Señalar con X los que procedan)

\begin{tabular}{|c|c|c|c|c|}
\hline $\begin{array}{c}\text { 12-14 años } \\
\left(1^{\circ} \text { y } 2^{\circ} \text { de ESO }\right)\end{array}$ & $\begin{array}{c}\text { 15-16 años } \\
\left(3^{\circ} \text { y } 4^{\circ} \text { de ESO }\right)\end{array}$ & $\begin{array}{l}\square \quad \mathbf{1 7 - 1 8} \text { años } \\
\text { (Bachillerato, Ciclos Formativos } \\
\text { Grado Medio) }\end{array}$ & $\begin{array}{l}\square 19 \text { o más años } \\
\text { (Ciclos } \quad \text { Formativos } \\
\text { Superior; Universidad) }\end{array}$ & Grado \\
\hline
\end{tabular}

3. Con la elección de carrera que hiciste en su día, te sientes: (Señalar con X)

\begin{tabular}{|c|c|c|c|}
\multicolumn{2}{c}{ MUY SATISFECH@ } \\
\hline 1 & 2 & 3 & 4 \\
\hline
\end{tabular}

4. Siempre que pudiste elegir las asignaturas optativas, lo hiciste en función de: (Señalar con $X$ las que procedan)
$\square$ Relación con la especialidad o trabajo futuro.
$\square$ Elección de tus compañeros.
No existe optatividad en mis estudios.
5. El Practicum, Proyecto de fin de carrera o Prácticas voluntarias en empresas, las has elegido o piensas elegirlas en función de: (Señalar con X un MÁXIMO DE TRES)
$\square$ Horario y proximidad a casa.
$\square$ Buenas referencias de la plaza o empresa.
$\square$ Interés por las tareas que allí se realizan.
$\square$ Orientación de tu tutor|@ de prácticas.
$\square$ Posibilidades de futuras salidas profesionales.
$\square$ Especialidad a la que quieres dedicarte.
$\square$ No tuviste posibilidad de elección.
$\square$ En mis estudios no existe practicum, prácticas, ....
$\square$ No he pensado en ello.
$\square$ No tengo claro qué elegir.

6. En este momento de la carrera, respecto a la especialidad o área de trabajo que buscas, tu situación es: (Señalar con $X$ y escribir lo que proceda)

$\square$ Tengo muy claro que lo que quiero hacer es:

$\square$ Dudo entre dos especialidades: A )

$\square$ Dudo entre tres especialidades.

$\square$ No tengo decidido nada.

у B)...

7. Respecto a experiencias de trabajo: (Señala con X la que mejor responda a tu situación actual):

$\square$ Ahora no trabajo, pero si he tenido experiencias laborales. $\square$ Estoy trabando en algo relacionado con lo que estudio.

$\square$ Estoy trabajando en algo, pero no está relacionado con lo $\square$ No he trabajado nunca.

que estudio.

\section{Cuando finalices los estudios tienes previsto hacer inmediatamente: (Señalar con $X$ un} MÁXIMO de TRES)

$\square$ Ejercer la profesión.

$\square$ Solicitar beca.

$\square$ No lo he decidido.

\author{
$\square$ Hacer un master o curso de postgrado. \\ $\square$ Hacer oposiciones..... \\ $\square$ Otra carrera ¿cuál?:.
}

\author{
$\square$ Hacer el doctorado. \\ $\square$ Buscar un trabajo cualquiera. \\ $\square$ No he pensado en ello.
}

9. Las dificultades o problemas que tienes para decidirte por una especialidad o área de trabajo, se concretan en: (Señalar con $X$ las que respondan a tu caso)
$\square$ Ninguna opción me satisface plenamente.
$\square$ No tengo claro la opción profesional que más me conviene.
$\square$ Me falta información fiable sobre mi mismo.
$\square$ Que mis preferencias profesionales cambien en el futuro.
$\square$ Desconozco qué es importante tener en cuenta para decidir adecuadamente.
$\square$ Personas importantes para mí, no apoyan mis planes.
$\square$ No tengo dificultades personales de decisión.
$\square$ No tengo contacto
trabajo que quiero. especialidad.
$\square$ Tengo la información académico-profesional que me hace falta. 
Esperanza Rocabert Beut et al.

[Página en blanco por motivos de paginación] 